\title{
Mild and eco-friendly chemoselective acylation of amines in aqueous medium
}

\author{
Sarala Naik, Gitalee Bhattacharjya, Veerababu Rao Kavala, and Bhisma K. Patel* \\ Department of Chemistry, Indian Institute of Technology, Guwahati-781 039, India \\ E-mail: patel@,iitg.ernet.in
}

(received 01 Oct 03; accepted 19 Apr 04; published on the web 24 Apr 04)

\begin{abstract}
Amines in the form of amine hydrochlorides are efficiently acylated with anhydrides in an aqueous medium on addition of $\mathrm{NaHCO}_{3}$. Both cyclic and acyclic anhydrides react with equal ease with an amine and amines of various stereo-electronic factors react with the same rates with an anhydride. No chromatographic separation is needed for isolation of the acylated product. Reactions in aqueous medium, innocuous by-products and chemoselective acylation of amines in the presence of phenols and thiols have been achieved with high selectivity.
\end{abstract}

Keywords: Amines, amides, acylation, anhydrides, aqueous medium, chemoselective

\section{Introduction}

The acylation of amines is a common and often used as a protecting group in organic synthesis as it provides an efficient and inexpensive means for protecting an amino functionality in a multi-step synthetic process. ${ }^{1}$ Acetic anhydride and acetyl chloride are generally used in the presence of acidic $^{2}$ or basic $^{3}$ catalysts in an organic medium. These reactions have advantages and drawbacks, recently described by Katritzky. ${ }^{4}$ Some of these reagents and catalysts lead to waste as well as some reactions involving organic solvents, often toxic and polluting, hence unacceptable in the present days. One of the major factors for a green chemical process in solution involves the choice of cheap, safe and non-toxic solvents. Water being abundant in nature is the first choice. In addition to satisfying the above criteria it has also special effects on reaction arising from intra- and inter- molecular non-covalent interactions leading to assembly processes. After Breslow's discovery of positive effect on the reaction rates and selectivities of Diels-Alder reaction, which is otherwise insensitive to solvent effects, special attention was focused on the origin of the aqueous acceleration. ${ }^{5,6}$ Thus, development of an efficient and convenient synthetic methodology in water is an important area of research. Considering the importance of acylation and environmental factors as well as our interest in green chemical processes, ${ }^{7}$ we report in this letter acylation of amines in an aqueous medium, which fulfils many of the above requirements. 


\section{Results and Discussion}

Acetylation of aromatic amines has been carried out in aqueous media but with a limited number of substrates. ${ }^{8}$ The same has been achieved using amine, hydrochloric acid, concentrated solution of sodium acetate $(5 \mathrm{M})$ and acetic anhydride. ${ }^{9}$ We thought to add sodium bicarbonate to an aqueous solution of amine hydrochloride, which will liberate free amine and react with acetic anhydride and convert the liberated acetic acid to sodium acetate in the medium. Aliphatic and aromatic amines are basic in nature and can easily be protonated by mineral acids. To test our hypothesis and to optimise the reaction conditions, aniline $\mathbf{4}$ was converted to water-soluble anilinium hydrochloride using aqueous $\mathrm{HCl}$. The protonated ammonium species is nonnucleophilic due to non-availability of the lone pair of electrons on the nitrogen atom. Thus, when acetic anhydride was added to an aqueous solution of amine hydrochloride no acetylation occurred. However, upon addition of basic salts, such as $\mathrm{NaHCO}_{3}$ to the above medium, free amines were liberated, which reacted immediately with acetic anhydride, precipitating the acetylated product with the evolution of carbon dioxide. The reaction works best when the final $\mathrm{pH}$ of the medium is ca. 5.5, approximately one $\mathrm{pKa}$ unit higher compared to that of acetic acid (pKa 4.8). Protonation of amine in an acidic medium has been confirmed by hypochromic shifts at $226 \mathrm{~nm}$ and $276 \mathrm{~nm}$ for $\left(\pi-\pi^{*}\right)$ and $\left(\mathrm{n}-\pi^{*}\right)$ respectively, by titrating a dilute solution of 2fluoroaniline 5 with a dilute solution of $\mathrm{HCl}$ using $\mathrm{UV}$ spectrophotometer. A hyperchromic shift of these transitions upon addition of a dilute solution of sodium bicarbonate confirms the regeneration of free amines.

As shown in Table 1 several amines underwent acetylation very smoothly in good yields. The optimised acetylation reaction was performed by adding acetic anhydride (1.5 equiv) to the substrate amine hydrochloride ( $\mathrm{pH}$ ca.1.6) dissolved in water followed by addition of $\mathrm{NaHCO}_{3}$ in one lot to obtain a final $\mathrm{pH}$ ca. 5.5. The methodology works well for both aliphatic, 1-2 and aromatic, 3-9 amines, as reported in Table $1 .{ }^{10}$ Primary amines of varying electronic and steric factors, substrate 3-9 were examined. It is interesting to note that in most of these cases the product precipitates in less than 5 minutes. It has been observed that the acetylation of aryl amines performed in an organic reaction medium, substrate containing electron-donating groups in the aromatic ring facilitate the reaction, whereas electron-withdrawing groups slow down the reaction. No such effect was observed by the present methodology and all the substrates react with equal rates. However, aryl amines gave better yields as compared to alkyl amines. Chiral amines 10 can be easily acetylated with complete retention of optical activity. Phenol and thiophenol reacted slowly under the identical conditions giving poor yields. Thus, by taking advantage of the differential reactivity between nucleophiles, we were able to carry out chemoselective acetylation of amines over phenols and thiols. Thus, in a competitive acetylation reaction with an equimolar mixture of aniline 4 and phenol by this procedure, the amine is acetylated selectively leaving the phenol unaffected. 
Table 1. Acetylation of amines with acetic anhydride

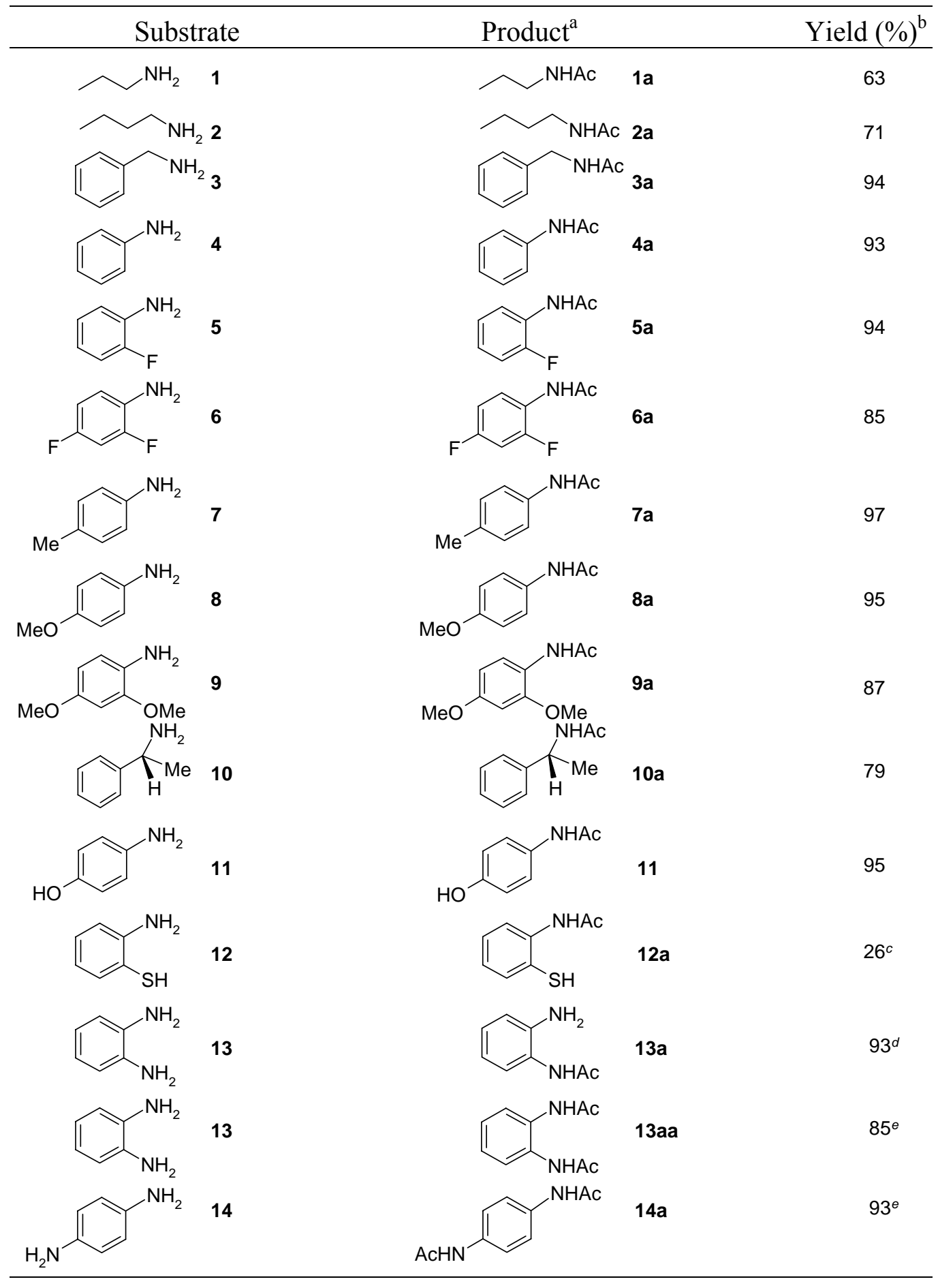

${ }^{\mathrm{a}}$ Confirmed by comparison with IR, ${ }^{1} \mathrm{H}$ and ${ }^{13} \mathrm{C}$ NMR of the authentic sample. ${ }^{\mathrm{b}}$ Isolated yields.

${ }^{\mathrm{c}}$ Rest of the products being diacetylated product 12aa and 2-methyl-benzothiazole 12ab. ${ }^{\mathrm{d}}$ Based on the recovery of starting material. ${ }^{\mathrm{e}} 3$ equivalent of $\mathrm{Ac}_{2} \mathrm{O}$ was used. 
In an analogous reaction between aniline $\mathbf{4}$ and thiophenol, the thiophenol remains unaffected. Similar selectivity was observed for intramolecular reaction as well. Thus, acetylation of 4-aminophenol 11 and 2-aminothiophenol 12 produced the corresponding acetamides; the phenolic and thiophenolic moiety remained untouched with one equivalent of the reagent. The selective acetylation is of significant interest for the preparation of antipyretic and analgesic drugs paracetamol 11a. Monoacetylation of 1,2-phenylenediamine 13 demonstrates the efficacy of the method. However, no chemoselectivity was observed for symmetrical diamine 1,4-phenylenediamine 14 even with one equivalent of the acetic anhydride.

When 2-aminothiophenol 12 was treated with one equivalent of acetic anhydride under the identical conditions; along with a trace amount of diacetylated product 12aa $(<5 \%)$ and monoacetylated product 12a $(>28 \%)$ and an interesting heterocyclic product 2-methylbenzothiazole 12ab was obtained (ca.40\%). The formation of 2-methyl-benzothiazole 12ab is via acetylation of amine followed by a nucleophilic attack of thiophenolic group on the carbonyl carbon of the amide and subsequent water elimination. Formation of 2-methyl-benzothiazole 12ab confirmed the chemoselective acetylation of amines over thiols. In most of these cases the acetylated product precipitated out from the aqueous reaction medium and in few cases it was extracted with ethyl acetate to yield the pure product, except in the case of substrates 1 and 2 the product required extraction and in the case of substrates $\mathbf{1 3}$ and $\mathbf{1 4}$ the products required extraction as well as chromatographic separation. It was found that primary amines underwent smooth acetylation where as secondary amines such as diphenylamine remained inert under the present experimental conditions. The by-product sodium acetate is a useful buffering agent and can be recovered from the aqueous effluent by concentrating the aqueous medium, if desired.

The novel aspect of the present methodology was applied to cyclic anhydride such as succinic and maleic anhydride. In this case the anhydride 1.2 equivalent was used per equivalent of amine. All the substrate reacts easily as shown in Table 2, giving good yields. The reaction took place readily with simultaneous precipitation of white solid product. Finally the methodology was tested with an aromatic cyclic anhydride, phthalic anhydride. Finely powdered phthalic anhydride, 1 equivalent, was added to the amine hydrochloride solution followed by solid sodium bicarbonate. This has been tested with a number of aromatic amines and the result is summarised in Table 2. 
Table 2. Acylation of amines with different anhydrides

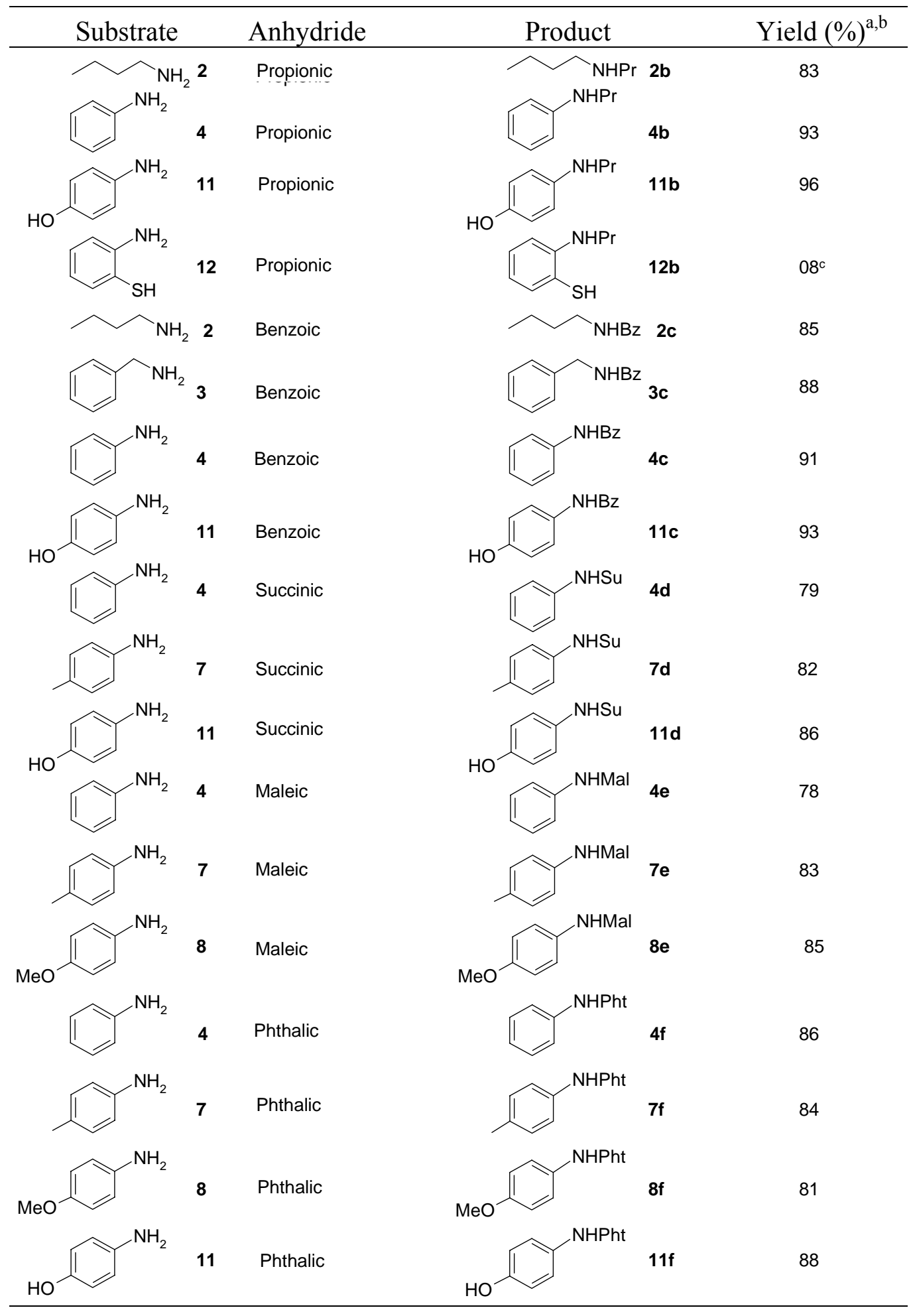

${ }^{a}$ Confirmed by comparison with IR, ${ }^{1} \mathrm{H}$ and ${ }^{13} \mathrm{C}$ NMR of the authentic sample. ${ }^{\mathrm{b}}$ Isolated yields.

c Rest of the products being 2-ethyl-benzothiazole 12ab (50\%). $\operatorname{Pr}=$ Propionyl; $\mathrm{Su}=\mathrm{COCH}_{2} \mathrm{CH}_{2} \mathrm{COOH} ; \mathrm{Mal}=\mathrm{COCH}=\mathrm{CHCOOH} ; \mathrm{Pht}=\mathrm{COC}_{6} \mathrm{H}_{5} \mathrm{COOH}$. 
Besides acetic anhydride this method is also amenable to acyclic, cyclic, aliphatic and aromatic anhydrides, Table 2. ${ }^{10}$ Propionylation of aliphatic 2 and aromatic amine $\mathbf{4}$ using propionic anhydride was carried out under the identical conditions as described for acetic anhydride. Here again the amino functionality has been chemoselectively propionylated in the presence of phenols and thiols as demonstrated for 4-aminophenol 11 and 2-aminothiol 12 respectively. Substrate 2-aminothiol 12 gave heterocyclic product 2-ethylbenzothiazole 12bb as the exclusive product and N-(2-mercapto-phenyl)-propionamide 12b as the minor product thereby supporting the chemoselective propionylation of amines over thiols. Benzoylation of aliphatic amine 2, benzylamine 3, aromatic amine 4 and chemoselective benzoylation of 4aminophenol 11 further proves the efficacy and chemoselectivity of the present aqueous methodology. In this case benzoic anhydride ( 1 equivalent) was added to an amine hydrochloride solution followed by solid sodium bicarbonate. The product precipitated into lumps, which can be recrystallised either from acetonitrile or from ethyl acetate. The by-product sodium benzoate can be recovered from the aqueous medium if desired.

\section{Conclusions}

In conclusion, this method represents a tremendous opportunity for the practice of green chemistry. The notable advantages of the method are: (i) operational simplicity, (ii) moderate to good yields, (iii) no chromatographic separation, (iv) excellent selectivity for aryl amines over phenols and thiols and (v) general applicability. The method is environmentally friendly with respect to by-products and the effluent are innocuous. The by-product sodium acetate / benzoate is a useful buffering agent. We believe this will present a better and more practical alternative to the existing methodologies for selective acylation of primary amines and thus will find useful application in the synthesis of complex natural products where selective protection of hydroxy, thio and amino groups is required.

\section{Experimental Section}

Typical Experimental Procedure. To a stirred heterogeneous suspension of amine (1 mmol) in water $(5 \mathrm{~mL}$ ) was added $6 \mathrm{~N} \mathrm{HCl}$ (in the volume range of $240-400 \mu \mathrm{L}$ ) until the solution became homogeneous ( $\mathrm{pH}$ ca.1.5). The resulting homogenous solution was cooled in an ice bath. To this was then added anhydride (1-1.5 mmol) followed by solid sodium bicarbonate (185-300 $\mathrm{mg})$ until there was no further effervescence or $\mathrm{pH}$ of the mixture becomes ca 5.5. The precipitate product was filtered, washed with water $(2 \times 1 \mathrm{~mL})$, dried by pressing between folds of filter paper and finally dried in a vacuum desiccator. In cases, where product did not precipitate out the reaction mixture was extracted with ethyl acetate $(2 \times 10 \mathrm{~mL})$. The organic extract was dried over anhydrous $\mathrm{Na}_{2} \mathrm{SO}_{4}$ and the solvent was evaporated in a rotary evaporator under reduced pressure to yield the pure product which were identified by comparison of their NMR, IR, GC and GC co-injection with authentic samples prepared by known methods. 


\section{Acknowledgements}

B.K.P. acknowledges the support of this research from DST New Delhi SP/S1/G-28/98 and CSIR 01(1688)/00/EMR-II; S.N acknowledges the financial support to the DST and G.B and V.B to the institute. Thanks are due to RSIC, Lucknow; DRDE Gwalior; and IIT Kanpur for providing NMR spectra.

\section{References and Notes}

1. (a) Greene, T. W.; Wuts, P. G. M. Protective Groups in Organic Synthesis, $3^{\text {rd }}$ Edn; Wiley \& Sons: New York, 1999. (b) Kocieński, P. J. Protecting Groups; Georg Thieme Verlag: New York, 1994.

2. (a) Chen, C.-T.; Kuo, J.-H.; Li, C.-H.; Barhate, N. B.; Hon, S.-W.; Li, T.-W.; Chao, S. -D.; Liu C.-C.; Li, Y.-C.; Chang, I.-H.; Lin, J.-S.; Liu, C.-J.; Chou, Y.-C. Org. Lett. 2001, 3, 3729. (b) Procopiou, P. A.; Baugh, S. P. D.; Flack, S. S.; Inglis, G. G. A. J. Org. Chem. 1998, 63, 2342. (c) Saravanan, P.; Singh. V. K. Tetrahedron Lett. 1999, 40, 2611. (d) Chauhan, K. K.; Frost, C. G.; Love, I.; Waite, D. Synlett 1999, 1743. (e) Zhou, H.; Pendri, A.; Greenwald, R. B. J. Org. Chem. 1998, 63, 7559. (f) Bhaskar, P. M.; Loganathan, D. Tetrahedron Lett. 1998, 39, 2215. (g) Li, T.-S.; Li, A. -X. J. Chem. Soc. Perkin Trans. 1 1998, 1913. (h) Chavan, S. P.; Anand, R.; Pasupathy, K.; Rao, B. S. Green Chemistry 2001, 3, 320. (i) Kumar, P.; Pandey, R. K.; Bodas, M. S.; Dangare, M. K. Synlett 2001, 206. (j) Sabitha, G.; Reddy, B. V. S.; Srividya, R.; Yadav, J. S. Synth. Commun. 1999, 29, 2311. (k) Orita, A.; Tanahashi, C.; Kakuda, A.; Otera, J. Angew. Chem., Int. Ed. 2000, 39, 2877.

3. (a) Vedejs, E.; Diver, S. T. J. Am. Chem. Soc. 1993, 115, 3358. (b) Vedejs, E.; Daugulis, O. J. Org. Chem. 1996, 61, 5702. (c) D’Sa, B. A.; Verkade, J. G. J. Org. Chem. 1996, 61, 2963. (d)Yadav, V. K.; Babu, K. G.; Mittal, M. Tetrahedron 2001, 57, 7047. (e) Paul, S.; Nanda, P.; Gupta, R.; Laupy, A. Tetrahedron Lett. 2002, 43, 4261.

4. Katritzky, A. R.; He, H. -Y.; Suzuki, K. J. Org. Chem. 2000, 65, 8210.

5. Rideout, D. C.; Breslow, R. J. Am. Chem. Soc. 1980, 102, 7816.

6. (a) Breslow, R. Acc. Chem. Res. 1991, 24, 159. (b) Li, C. -J. Chem. Rev. 1993, 93, 2023. (c) Herrmann, W. A.; Kohlpaintner, C. W. Angew. Chem., Int. Ed. 1993, 32, 1524. (d) Lubineau, A.; Auge, J.; Queneau, Y. Synthesis 1994, 741. (e) Li, C. -J. Tetrahedron 1996, 52, 5643. (f) Kobayashi, S. Synlett 1994, 689. (g) Lindström, U. M. Chem. Rev. 2002, 102, 2751. (h) Shu, K.; Kei, M. Acc. Chem. Res. 2002, 35, 209. (i) Okuhara, T. Chem. Rev. 2002, 102, 3641. (j) Pae, A. N.; Cho, Y. S. Current Organic Chemistry 2002, 6, 715.

7. (a) Gopinath, R.; Barkakaty, B.; Talukdar, B.; Patel, B. K. J. Org. Chem. 2003, 68, 2944. (b) Gopinath, R.; Haque, Sk. J.; Patel, B. K. J. Org. Chem. 2002, 67, 5842. (c) Gopinath, R.; Paital, A. R.; Patel, B. K. Tetrahedron Lett. 2002, 43, 5123. (d) Naik, S.; Gopinath, R.; Patel, 
B. K. Tetrahedron Lett. 2001, 42, 7679. (e) Gopinath, R.; Patel, B. K. Org. Lett. 2000, 2, 4177. (f) Gopinath, R.; Patel, B. K. Org. Lett. 2000, 2, 577.

8. (a) DeWitt, H. D.; Indersoll, A.W. J. Am. Chem. Soc. 1951, 73, 3359. (b) Roseman, S.; Ludowieg, J. J. Am. Chem. Soc. 1954, 76, 301. (c) Richardson, A. C.; McLauchlan, K. A. J. Chem. Soc. 1962, 2499. (d) Kim, D-H.; Rho, H-S.; You, J. W.; Lee, J. C. Tetrahedron Lett. 2002, 43, 277. (e) Srivastava, V.; Tandon, A.; Ray, S. Synth. Commun. 1992, 22, 2703. (f) Sakakibara, T.; Watabe, Y.; Yamada, M.; Sudoh, R. Bull. Chem. Soc. Jpn. 1988, 61, 247.

9. Furniss, B. S.; Hannaford, A. J.; Smith, P. W. G.; Tatchell, A. R. Vogel's Textbook of Practical Organic Chemistry. $5^{\text {th }}$ Edn.; Longman: Singapore, 1989.

10. Selected spectroscopic data: $\boldsymbol{N}$-(2-fluoro-phenyl)-acetamide (5a): ${ }^{1} \mathrm{H}$ NMR (400 MHz, $\left.\mathrm{CDCl}_{3}\right) \delta 2.2(\mathrm{~s}, 3 \mathrm{H}), 7.02(\mathrm{~m}, 1 \mathrm{H}), 7.28(\mathrm{~s}, 1 \mathrm{H}), 7.3(\mathrm{~s}, 1 \mathrm{H}), 7.42(\mathrm{~s}, 1 \mathrm{H}), 8.2(\mathrm{~m}, 1 \mathrm{H}) ;{ }^{13} \mathrm{C}$ NMR $\left(100 \mathrm{MHz}, \mathrm{CDCl}_{3}\right) \delta 24.45,114.64,114.89,122.17,124.35,124.38,124.43,126.17$, 126.31, 150.91, 154.13, 168.63. $\mathrm{N}$-(2,4-Difluoro-phenyl)-acetamide (6a): ${ }^{1} \mathrm{H}$ NMR (400 $\left.\mathrm{MHz} \mathrm{CDCl}_{3}\right) \delta 2.20(\mathrm{~s}, 3 \mathrm{H}), 6.86(\mathrm{~m}, 2 \mathrm{H}), 7.27$ (brs, 1H), $8.22(\mathrm{~m}, 1 \mathrm{H}) ;{ }^{13} \mathrm{C}$ NMR $(100$ $\left.\mathrm{MHz}, \mathrm{CDCl}_{3}\right) \delta 24.50,103.25,103.48,103.52,103.76,111.10,111.14,111.32,111.35$, 122.52, 122.91, 123.00, 153.56, 157.36, 159.81, 168.28. $\quad N$-(2,4-Dimethoxy-phenyl)acetamide (9a): ${ }^{1} \mathrm{H}$ NMR $\left(400 \mathrm{MHz}, \mathrm{CDCl}_{3}\right) \delta 2.15(\mathrm{~s}, 3 \mathrm{H}), 3.77(\mathrm{~s}, 3 \mathrm{H}), 3.82(\mathrm{~s}, 3 \mathrm{H}), 6.44$ $(\mathrm{m}, 2 \mathrm{H}), 7.52$ (brs, $1 \mathrm{H}), 8.18(\mathrm{~d}, 1 \mathrm{H}, J=9.5 \mathrm{~Hz}) ;{ }^{13} \mathrm{C} \mathrm{NMR}\left(100 \mathrm{MHz}, \mathrm{CDCl}_{3}\right) \delta 24.66$, 55.47, 55.60, 98.49, 103.65, 120.74, 121.18, 149.10, 156.27, 167.86. (R)- $N$-Phenyl-ethylacetamide (10a): ${ }^{1} \mathrm{H}$ NMR $\left(200 \mathrm{MHz}, \mathrm{DMSO}_{-} \mathrm{d}_{6}\right) \delta 1.30(\mathrm{~d}, 3 \mathrm{H}, J=7.0 \mathrm{~Hz}), 1.82(\mathrm{~s}, 3 \mathrm{H})$, $4.87(\mathrm{~m}, 1 \mathrm{H}), 7.23(\mathrm{~m}, 5 \mathrm{H}), 8.4$ (brs, $1 \mathrm{H}) ;{ }^{13} \mathrm{C}$ NMR $\left(50 \mathrm{MHz}, \mathrm{DMSO}-\mathrm{d}_{6}\right) \delta 22.78,22.96$, 48.15, 126.29, 126.92, 128.57, 145.09, 168.82. $\mathbf{N}$-(2-Mercapto-phenyl)-acetamide (12a): ${ }^{1} \mathrm{H}$ NMR $\left(300 \mathrm{MHz}, \mathrm{CDCl}_{3}\right) \delta 1.27(\mathrm{~s}, 1 \mathrm{H}), 1.98(\mathrm{~s}, 3 \mathrm{H}), 7.02(\mathrm{~m}, 1 \mathrm{H}), 7.40(\mathrm{~m}, 2 \mathrm{H}) 7.93$ (brs, 1H), $8.31(\mathrm{~m}, 1 \mathrm{H}) ;{ }^{13} \mathrm{C}$ NMR $\left(75 \mathrm{MHz}, \mathrm{CDCl}_{3}\right) \delta 24.97,121.75,123.47,124.40,124.88$, 132.48, 136.69, 140.19,168.91. Thioacetic acid $\boldsymbol{S}$-(2-acetylamino-phenyl)ester (12aa): ${ }^{1} \mathrm{H}$ NMR $\left(200 \mathrm{MHz}, \mathrm{CDCl}_{3}\right) \delta 2.14(\mathrm{~s}, 3 \mathrm{H}), 2.42(\mathrm{~s}, 3 \mathrm{H}), 7.13(\mathrm{~m}, 1 \mathrm{H}), 7.42(\mathrm{~m}, 2 \mathrm{H}), 7.69$ (brs, $1 \mathrm{H}), 8.25$ (brs, $1 \mathrm{H}) ;{ }^{13} \mathrm{C}$ NMR $\left(50 \mathrm{MHz}, \mathrm{CDCl}_{3}\right) \delta 25.02,30.70,117.73,122.86,125.20$, 131.91, 136.46, 140.02, 168.67, 193.82. 2-Methyl-benzothiazole (12ab): ${ }^{1} \mathrm{H}$ NMR (300 $\left.\mathrm{MHz}, \mathrm{CDCl}_{3}\right) \delta 2.81(\mathrm{~s}, 3 \mathrm{H}), 7.29(\mathrm{~m}, 1 \mathrm{H}), 7.43(\mathrm{~m}, 1 \mathrm{H}), 7.78(\mathrm{~m}, 1 \mathrm{H}), 7.93(\mathrm{~m}, 1 \mathrm{H}) ;{ }^{13} \mathrm{C}$ NMR $\left(75 \mathrm{MHz}, \mathrm{CDCl}_{3}\right) \delta 20.07,121.35,122.34,124.66,125.87,135.60,153.31,166.92 . \mathbf{N}-$ (2-Amino-phenyl)-acetamide (13a): ${ }^{1} \mathrm{H} \mathrm{NMR}\left(200 \mathrm{MHz}, \mathrm{CDCl}_{3}\right) \delta 2.10$ (s, 3H), 3.65 (brs, 2H), $6.75(\mathrm{~m}, 2 \mathrm{H}), 7.01(\mathrm{~m}, 2 \mathrm{H}), 7.64$ (brs, $1 \mathrm{H}) ;{ }^{13} \mathrm{C} \mathrm{NMR}\left(50 \mathrm{MHz}, \mathrm{CDCl}_{3}\right) \delta 23.94,118.41$, 119.81, 126.04, 127.72, 129.42, 141.37, 169.69. N-(2-Acetylamino-phenyl)-acetamide (13aa): ${ }^{1} \mathrm{H}$ NMR (300 MHz, DMSO-d 6 ) $\delta 2.07$ (s, 6H), $7.11(\mathrm{~m}, 2 \mathrm{H}), 7.52$ (m, 2H), 9.37 (s, $2 \mathrm{H}) ;{ }^{13} \mathrm{C}$ NMR (75 MHz, DMSO-d 6 ) $\delta$ 23.63, 124.51, 124.67, 130.40, 168.57. N-(4Acetylamino-phenyl)-acetamide (14a): ${ }^{1} \mathrm{H}$ NMR (300 MHz, DMSO-d $\left.\mathrm{d}_{6}\right) \delta 2.06(\mathrm{~s}, 6 \mathrm{H}), 7.10$ $(\mathrm{m}, 2 \mathrm{H}), 7.53(\mathrm{~m}, 2 \mathrm{H}), 9.43(\mathrm{~s}, 2 \mathrm{H}) ;{ }^{13} \mathrm{C}$ NMR (75 MHz, DMSO-d 6$) \delta 23.7,124.8,130.5$, 168.8. $\mathbf{N}$-(4-Hydroxy-phenyl)-propionamide (11b): ${ }^{1} \mathrm{H}$ NMR (400 MHz, DMSO-d $\left.{ }_{6}\right) \delta 1.04$ $(\mathrm{t}, 3 \mathrm{H}), 2.26(\mathrm{q}, 2 \mathrm{H}), 7.47(\mathrm{~s}, 4 \mathrm{H}), 9.76(\mathrm{~s}, 2 \mathrm{H}) ;{ }^{13} \mathrm{C}$ NMR $\left(100 \mathrm{MHz}, \mathrm{DMSO}-\mathrm{d}_{6}\right) \delta 9.86$, 18.57, 114.99, 120.80, 131.08, 153.05, 171.24. N-(2-Mercapto-phenyl)-propionamide 
(12b): ${ }^{1} \mathrm{H}$ NMR (400 MHz, $\left.\mathrm{CDCl}_{3}\right) \delta 1.10(\mathrm{t}, 3 \mathrm{H}), 2.14$ (q, 2H), $6.96(\mathrm{t}, 1 \mathrm{H}), 7.36(\mathrm{~m}, 3 \mathrm{H})$, $7.95(\mathrm{~s}, 1 \mathrm{H}), 8.33(\mathrm{~d}, 1 \mathrm{H}) ;{ }^{13} \mathrm{C}$ NMR $\left(100 \mathrm{MHz}, \mathrm{CDCl}_{3}\right) \delta .9 .49,30.62,120.85,124.10$, 132.03, 136.35, 139.79, 171.94. 2-Ethyl-benzothiazole (12bb): ${ }^{1} \mathrm{H}$ NMR (400 MHz, $\left.\mathrm{CDCl}_{3}\right)$ $\delta 1.47(\mathrm{t}, 3 \mathrm{H}), 3.15(\mathrm{q}, 2 \mathrm{H}), 7.25(\mathrm{t}, 1 \mathrm{H}), 7.35(\mathrm{t}, 1 \mathrm{H}), 7.75(\mathrm{~d}, 1 \mathrm{H}), 7.88(\mathrm{~d}, 1 \mathrm{H}) ;{ }^{13} \mathrm{C}$ NMR $\left(100 \mathrm{MHz}, \mathrm{CDCl}_{3}\right) \delta$ 13.76, 27.65, 121.45, 122.34, 124.61, 125.87, 134.90, 152.99, 173.72. $N$ - (4-Hydroxy-phenyl)-benzamide (11c): ${ }^{1} \mathrm{H}$ NMR (400 MHz, DMSO-d $\left.{ }_{6}\right) \delta 6.75$ (m, $\left.2 \mathrm{H}\right)$, 7.38-7.61(m, 6H), $7.93(\mathrm{~m}, 2 \mathrm{H}), 10.04(\mathrm{~s}, 1 \mathrm{H}) ;{ }^{13} \mathrm{C}$ NMR (100 MHz, DMSO-d 6 ) $\delta 115.05$, $122.35,127.56,127.74,128.34,131.31,135.23,153.86,165.03 . N$-p-Tolyl-succinamic acid (7d): ${ }^{1} \mathrm{H}$ NMR (400 MHz, DMSO-d 6 ) $\delta 2.19$ (s, 3H), 2.49 (s, 4H), 7.04 (d, $2 \mathrm{H}, J=8.4 \mathrm{~Hz}$ ), $7.42(\mathrm{~d}, 2 \mathrm{H}, J=8.4 \mathrm{~Hz}), 9.82(\mathrm{~s}, 1 \mathrm{H}), 12.08($ brs, $1 \mathrm{H}) ;{ }^{13} \mathrm{C}$ NMR $(100 \mathrm{MHz}$, DMSO-d 6 ) $\delta$ 20.44, 28.84, 30.71, 118.92, 129.06, 131.77, 136.84, 169.83, 173.91. $N$ - (4-Hydroxyphenyl)-succinamic acid (11d): ${ }^{1} \mathrm{H}$ NMR $\left(300 \mathrm{MHz}, \mathrm{DMSO}^{\left.-\mathrm{d}_{6}\right)} \delta 2.56(\mathrm{~s}, 4 \mathrm{H}), 6.74(\mathrm{~d}\right.$, $2 \mathrm{H}, J=8.7 \mathrm{~Hz}), 6.90(\mathrm{~m}, 1 \mathrm{H}), 7.20(\mathrm{~m}, 1 \mathrm{H}), 7.41(\mathrm{~d}, 2 \mathrm{H}, J=8.4 \mathrm{~Hz}), 9.74(\mathrm{~s}, 1 \mathrm{H}) ;{ }^{13} \mathrm{C}$ NMR $\left(75 \mathrm{MHz}, \mathrm{DMSO}-\mathrm{d}_{6}\right) \delta 29.2,31.1,116.1,121.1,131.2,153.3,169.8,174.2$. N-p-Tolylphthalamic acid (7f): ${ }^{1} \mathrm{H}$ NMR $\left(300 \mathrm{MHz}, \mathrm{DMSO}_{-} \mathrm{d}_{6}\right) \delta 2.26(\mathrm{~s}, 3 \mathrm{H}), 7.13(\mathrm{~d}, 2 \mathrm{H}), 7.50-$ $7.67(\mathrm{~m}, 5 \mathrm{H}), 7.87(\mathrm{~d}, 1 \mathrm{H}), 10.25(\mathrm{~s}, 1 \mathrm{H}), 13.02(\mathrm{~s}, 1 \mathrm{H}) ;{ }^{13} \mathrm{C}$ NMR $\left(75 \mathrm{MHz}, \mathrm{DMSO}-\mathrm{d}_{6}\right) \delta$ 20.68, 119.14, 127.31, 128.50, 128.83, 129.02, 129.56, 131.14, 131.73, 136.56, 138.41, 166.48, 166.86. $\mathrm{N}$-(4-Methoxy-phenyl)-phthalamic acid (8f) : ${ }^{1} \mathrm{H}$ NMR $(300 \mathrm{MHz}$, DMSO-d $\left._{6}\right) \delta 3.75(\mathrm{~s}, 3 \mathrm{H}), 6.92(\mathrm{~d}, 2 \mathrm{H}), 7.55-7.67(\mathrm{~m}, 5 \mathrm{H}), 7.87(\mathrm{~d}, 1 \mathrm{H}), 10.32(\mathrm{~s}, 1 \mathrm{H}) .13 .10$ $(\mathrm{s}, 1 \mathrm{H}) ;{ }^{13} \mathrm{C}$ NMR $(75 \mathrm{MHz}$, DMSO-d 6 ) $\delta 55.22,113.46,120.78,127.39,128.88,129.10$, 129.74, 131.19, 132.35, 138.51, 154.81, 166.36, 167.02. $\boldsymbol{N}$-(4-Hydroxy-phenyl)-phthalamic acid (11f): ${ }^{1} \mathrm{H}$ NMR (300 MHz, DMSO-d $\left.\mathrm{d}_{6}\right) \delta 6.72(\mathrm{~d}, 2 \mathrm{H}), 7.44-7.66(\mathrm{~m}, 5 \mathrm{H}), 7.84(\mathrm{~d}, 1 \mathrm{H})$, $9.20(\mathrm{~s}, 1 \mathrm{H}), 10.07(\mathrm{~s}, 1 \mathrm{H}), 13.14(\mathrm{~s}, 1 \mathrm{H}) ;{ }^{13} \mathrm{C}$ NMR $\left(75 \mathrm{MHz}, \mathrm{DMSO}-\mathrm{d}_{6}\right) \delta 114.49,120.79$, $127.24,128.64,128.86,129.69,130.69,130.93,138.37,152.70,165.91,166.88$. 